\title{
PHASIC ABERRANT VENTRICULAR CONDUCTION
}

\author{
BY
}

\section{SCHAMROTH AND E. CHESLER}

From the Baragwanath Hospital and University of the Witwatersrand, Johannesburg, South Africa

Received July 31, 1962

\begin{abstract}
"Thus the spread of the excitation wave to the ventricles, considered together, is abnormal by reason of a defect in a chief distributing channel; consequently I term the resultant contractions aberrant, for they are the product of impulses which have gone astray."
\end{abstract}

Thomas Lewis

A bizarre QRS complex is not necessarily due to an ectopic ventricular discharge but may be due to abnormal intraventricular conduction of a supraventricular impulse. Lewis (1912) termed this condition aberrant ventricular conduction. This definition may be applied to both permanent and temporary forms of left and right bundle-branch block, the conduction abnormality of the Wolff-Parkinson-White syndrome, and peri-infarction block. In modern usage, however, the term has come to be associated with the temporary, intermittent, or isolated forms of abnormal intraventricular conduction. We propose the term phasic aberrant ventricular conduction for this temporary form to distinguish it from the permanent forms of aberrant ventricular conduction which we term non-phasic aberrant ventricular conduction.

Aberrant ventricular conduction results in a bizarre QRS complex resembling left or right bundle-branch block (the exception to this is peri-infarction block where the QRS complexes are not bizarre). In phasic aberrant ventricular conduction these bizarre QRS complexes occur during rhythms that otherwise show normal intraventricular conduction. Thus, phasic aberrant ventricular conduction may mimic the bizarre complexes found in ventricular ectopic rhythms, e.g. paroxysmal ventricular tachycardia. The recognition of phasic aberrant ventricular conduction is thus of major import as the differentiation of supraventricular from ventricular rhythms affects both prognosis and treatment. Despite the early recognition by Lewis (1910) and further investigation by Lewis and Master (1925), Scherf (1927), Gouaux and Ashman (1947), Langendorf (1950 and 1951), and Scherf and Schott (1953), this subject has not received sufficient emphasis although it constitutes one of the more important applications of electrocardiography to clinical medicine.

\section{Mechanism of Phasic Aberrant Ventricular Conduction}

Phasic aberrant ventricular conduction may occur with any supraventricular rhythm, namely sinus rhythm, A-V nodal rhythm, atrial and A-V nodal extrasystoles, paroxysmal atrial and A-V nodal tachycardias, atrial flutter and atrial fibrillation. This disturbance is dependent upon (1) unequal refractory periods of the bundle branches; (2) early impulse formation; or (3) the length of the preceding $\mathbf{R}-\mathbf{R}$ interval.

In the presence of equal refractory periods of the bundle branches (A of Fig. 1), an impulse falling at position 2 will find both bundle branches fully recovered and will be normally conducted. An impulse falling at position 1 will find both bundles refractory and will be blocked. In the presence of unequal refractory periods of the bundle branches (B of Fig. 1) an impulse falling at position 3 
will be normally conducted, and one falling at position 1 will be blocked; but an impulse falling at position 2, i.e. between positions 1 and 3 will find one bundle branch recovered and the other refractory: it will thus be conducted down one bundle branch only, resulting in the bizarre QRS pattern of bundle-branch block. It is usually the left bundle branch that recovers first and thus phasic aberrant ventricular conduction usually results in a right bundle-branch block pattern.
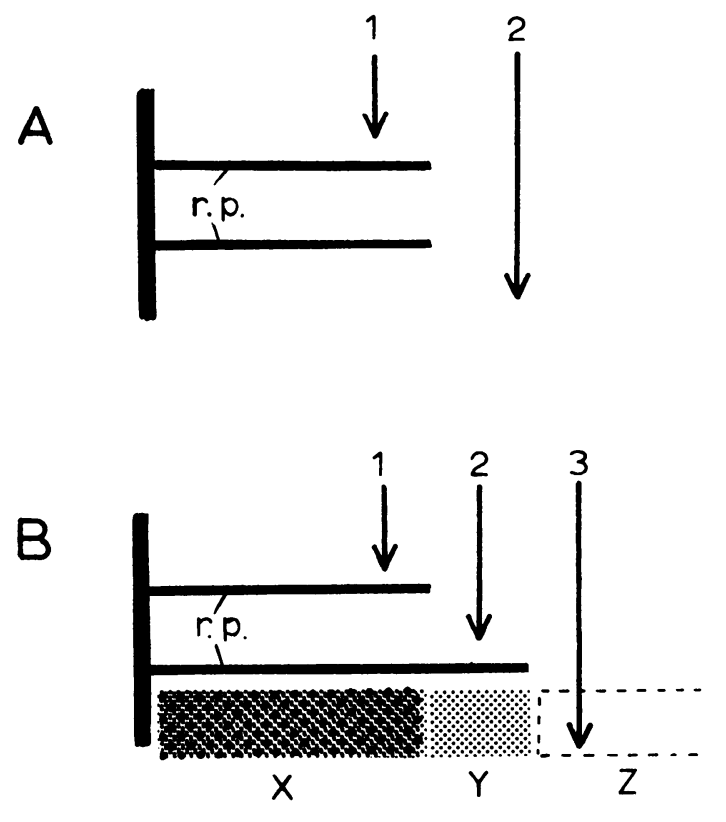

FIG. 1.-Diagrammatic representation of recovery of the bundle branches and the effect on subsequent impulse formation: (A) with equal refractory periods (r.p.) of the bundle branches. (B) with unequal refractory periods of the bundle branches. $\mathrm{X}=\mathrm{zone}$ of non-responsiveness; $Y=$ zone of unequal responsiveness; $Z=z o n e$ of equal responsiveness. Full explanation in text.

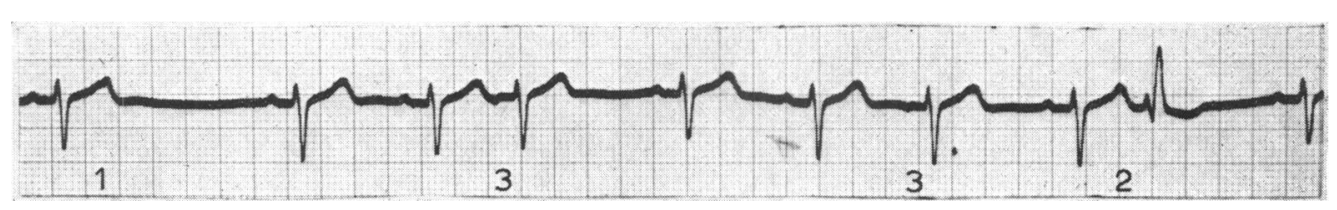

FIG. 2.-Electrocardiogram showing atrial extrasystoles. An extrasystole occurring very early in diastoleposition $1-$ is blocked (the $\mathbf{P}$ wave of the extrasystole is superimposed on the end of the $\mathbf{S}-\mathbf{T}$ segment); extrasystoles occurring relatively late-positions 3-are normally conducted; an extrasystole intermediate in timing, i.e. position 2 , is conducted with aberration (the $P$ wave of this extrasystole is superimposed upon and deforms the $T$ wave).

The presence of unequal refractory periods of the bundle branches divides the recovery period into three zones: (1) the zone of non-responsiveness (zone X in B of Fig. 1); (2) the zone of unequal responsiveness (zone $\mathrm{Y}$ in $\mathrm{B}$ of Fig. 1); and (3) that of equal responsiveness (zone $\mathrm{Z}$ in $\mathrm{B}$ of Fig. 1). Hence it will be seen that unequal refractory periods are essential to the occurrence of phasic aberrant ventricular conduction, and that the phenomenon is favoured by early or premature impulses-the earlier the impulse, within limits, the more likely it is to fall in the zone of unequal responsiveness of the bundle branches.

These phenomena are illustrated in Fig. 2 and 3. Fig. 2 shows atrial extrasystoles: very early extrasystoles are blocked, late extrasystoles are normally conducted, extrasystoles intermediate in timing are conducted with aberration. In Fig. 3 is shown an example of intermittent bundle-branch block dependent upon critical rate (Vesell, 1941; Shearn and Rytand, 1953). With a relatively slow heart rate, the sinus impulses find both bundle branches fully recovered and are normally conducted 


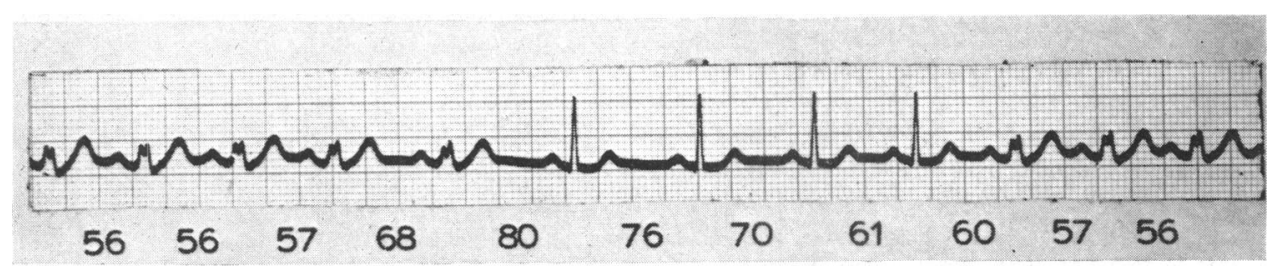

FIG. 3.-Electrocardiogram showing bundle-branch block with a relatively fast rate, and normal intraventricular conduction with a slower rate. Time intervals are indicated in hundredths of a second.

(mechanism illustrated by position 3 in B of Fig. 1). With an increase in heart rate, the sinus impulses find one bundle branch refractory and the other recovered; they are therefore conducted with the bundle-branch block pattern of phasic aberrant ventricular conduction (mechanism illustrated by position 2 in B of Fig. 1).

The duration of the refractory period is directly proportional to the length of the preceding $\mathbf{R}-\mathbf{R}$ interval (Fig. 4). With a long preceding $R-R$ interval the subsequent refractory period will be long (II in A of Fig. 4): with a short preceding $R-R$ interval the subsequent refractory period will be short (I in A of Fig. 4). In other words, the refractory period shortens with tachycardia and

FiG. 4.-Diagrammatic representation of the effect of
A

II

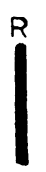

$\stackrel{R}{1}$
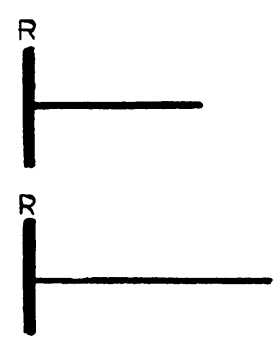

short and long $\mathbf{R}-\mathbf{R}$ intervals on the subsequent refractory periods. Full explanation in text.

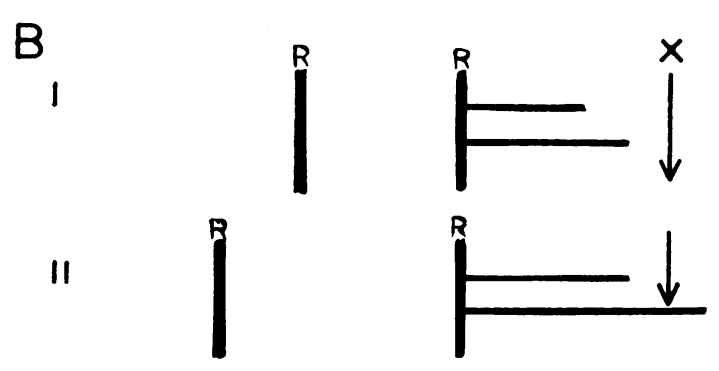

lengthens with bradycardia (Trendelenburg, 1903; Mines, 1913; Lewis, Drury, and Bulger, 1921). The same principle applies in the presence of unequal refractory periods of the bundle branches. With a short preceding $\mathbf{R}-\mathbf{R}$ interval the subsequent refractory periods of the bundle branches will be relatively short (I in B of Fig. 4); an early beat-X-will find both bundle branches recovered and will be conducted normally. With a long preceding $R-R$ interval the subsequent refractory periods of the bundle branches will be relatively long (II in B of Fig. 4) and an early impulse-X, at the same distance from the sinus beat as in B I-will now find one bundle branch refractory and be conducted with ventricular aberration. Phasic aberrant ventricular conduction is thus 
favoured by a long preceding $\mathrm{R}-\mathrm{R}$ interval. This is illustrated in Fig. 5. The tracing shows three atrial extrasystoles-A, B, and C. Extrasystoles A and B result in identical R-R intervals $(0.41$ sec.), yet extrasystole $A$ is conducted normally whereas extrasystole $B$ is conducted with ventricular aberration. This is because extrasystole $B$ is preceded by a long $R-R$ interval $(0.70$ sec.), whereas extrasystole $A$ is preceded by a short $R-R$ interval $(0.58 \mathrm{sec}$.).

This principle is also illustrated in Fig. 6 where only the second beat of a run of paroxysmal supraventricular tachycardia shows aberrant ventricular conduction, a pattern that may almost be termed a hallmark of aberrant ventricular conduction. This is because only the second beat of the paroxysm is preceded by a long $R-R$ interval, whereas the subsequent beats due to the tachycardia are preceded by short $\mathrm{R}-\mathrm{R}$ intervals. If, however, the second and subsequent beats occur very early, then all the beats except the first will show ventricular aberration (Fig. 7 and 8).

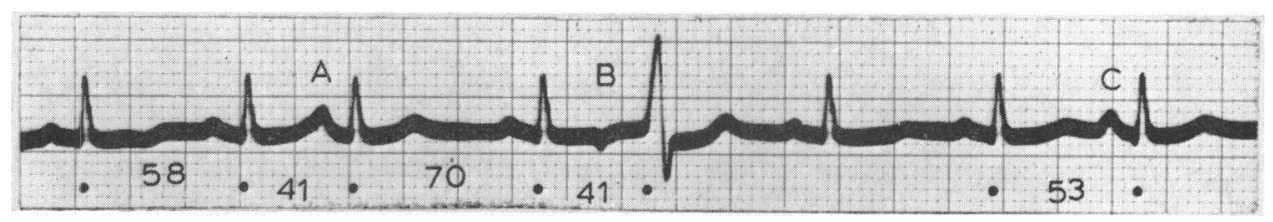

FIG. 5.-Electrocardiogram showing three atrial extrasystoles A, B, and C. Extrasystole C occurs relatively late and is thus normally conducted. Extrasystoles $\mathrm{A}$ and $\mathrm{B}$ result in identical $\mathbf{R}-\mathbf{R}$ intervals $(0.41 \mathrm{sec}$.); $A$ is normally conducted as it is preceded by a relatively short $R-R$ interval $(0.58 \mathrm{sec}$.); $\mathrm{B}$ is conducted with aberration as it is preceded by a relatively long $\mathrm{R}-\mathrm{R}$ interval $(0.70 \mathrm{sec}$.$) .$
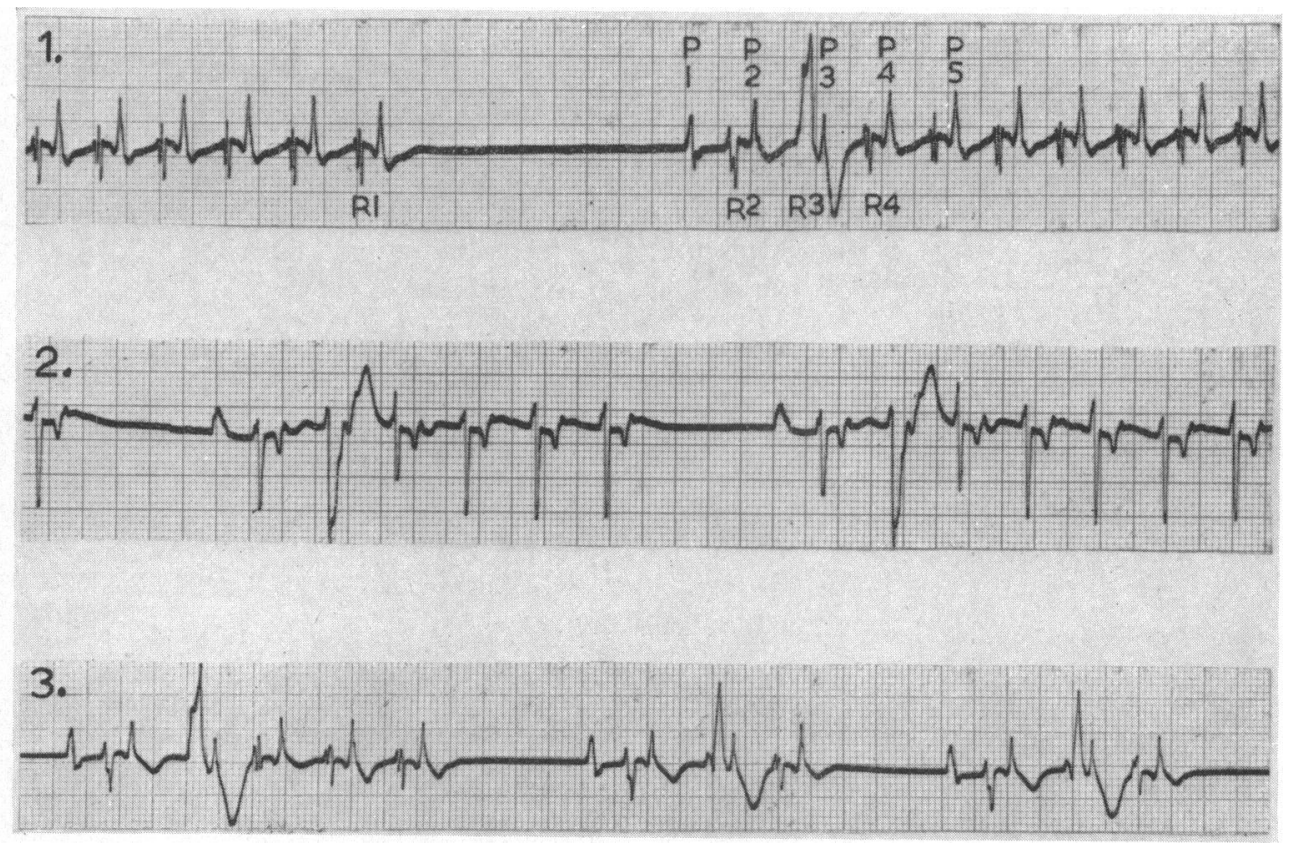

FIG. 6.-Electrocardiograms (strips 1 and 3, lead V1; strip 2, standard lead II) showing paroxysms of reversed reciprocating paroxysmal tachycardia (Schamroth, 1960). Note that only the second QRS complex of each paroxysm is conducted with aberration; this is because it is only the second atrial impulse of each paroxysm (e.g. P2 of strip 1) that is preceded by a long $R-R$ interval (e.g. R1-R2 of strip 1). 


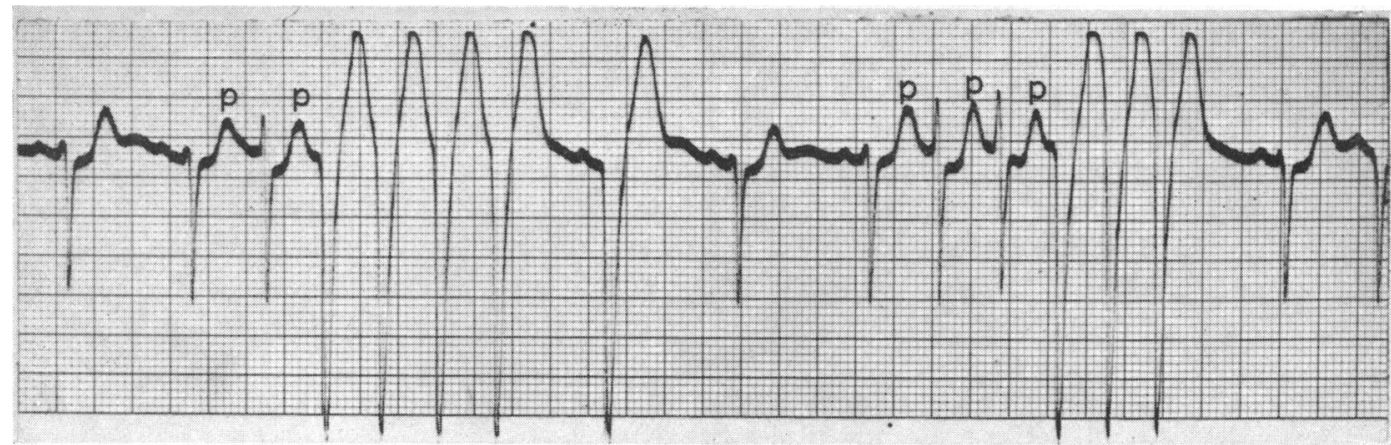

Fig. 7.-Electrocardiogram showing two paroxysms of atrial tachycardia with aberrant ventricular conduction. The $P$ waves (labelled) of the ectopic atrial rhythm are seen at the beginning of each paroxysm superimposed upon the T waves before the bizarre QRS complexes. Note the increasing abnormality of the QRS complexes as the rate speeds up.

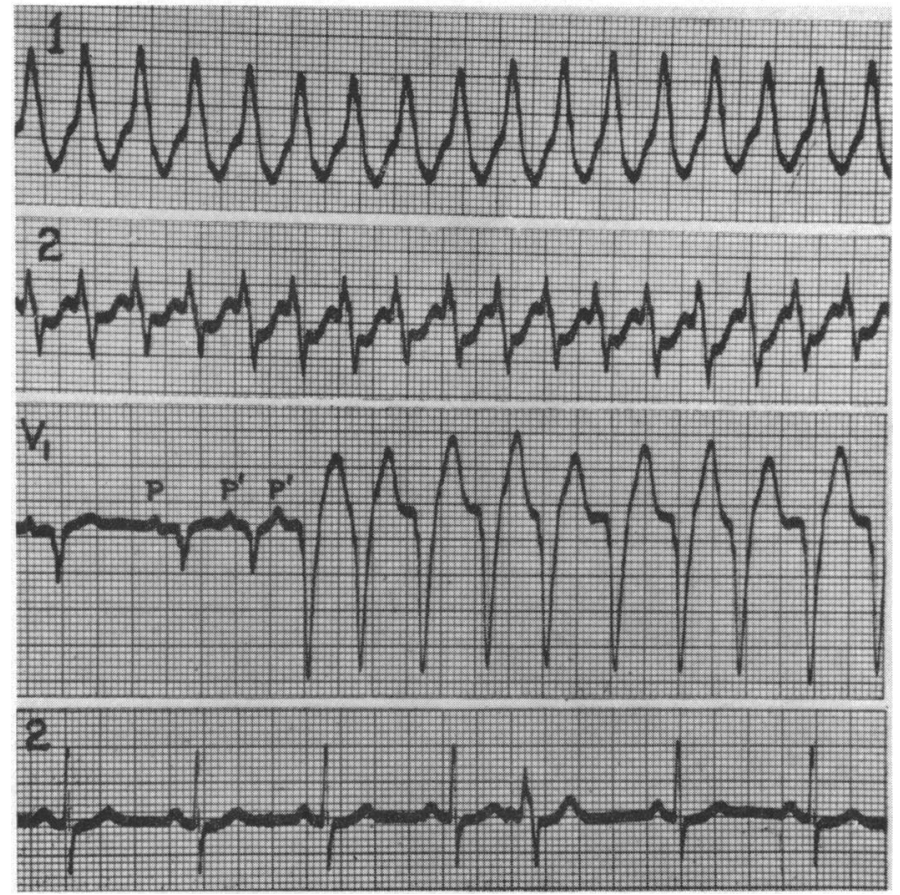

FIG. 8.-Electrocardiograms showing paroxysms of atrial tachycardia with aberrant ventricular conduction. The upper two rows (standard leads I and II) show what appears to be typical ventricular paroxysmal tachycardia. However, $\mathbf{P}$ waves cannot be identified. The true nature of this rhythm becomes apparent from further tracings. The third strip (lead V1) shows the beginning of the tachycardia. Note that the paroxysm commences with two premature ectopic $\mathbf{P}$ waves (labelled $\mathbf{P}^{\prime}$ ) the second of which is conducted with aberration. The lower strip (standard lead 2) shows an atrial extrasystole conducted with aberration; the resulting bizarre QRS complex has the same form as that of the bizarre QRS complexes during the tachycardia seen in the second strip. 


\section{Differentiation of Ectopic Ventricular Rhythms from Phasic Aberrant VENTRICULAR CONDUCTION}

Recognition of Phasic Aberrant Ventricular Conduction in Presence of Supraventricular Rhythms other than Atrial Fibrillation. For the diagnosis of phasic aberrant ventricular conduction there must be a $\mathbf{P}$ wave before and related to the bizarre QRS complex. For the diagnosis of ectopic ventricular rhythms it must be shown that the $P$ waves are completely dissociated from the QRS complexes or that their timing is such that they indicate retrograde conduction of the ectopic ventricular impulses. The presence of fusion beats and captures also indicates ectopic ventricular rhythm. These differentiating features are easily seen during slow rhythms or with isolated beats (Fig. 2, 3, 5, and Fig. 8, bottom strip). With fast rhythms, however, the differentiation of phasic aberrant ventricular conduction and ectopic ventricular rhythms may be extremely difficult and at times impossible. Under these circumstances the $P$ waves may be hidden in the QRS complexes and their relation to these QRS complexes obscured (Fig. 8, upper two strips). This diagnostic difficulty is well illustrated by Schrire and Vogelpoel (1955) who found that of 10 consecutive examples of eventually proven ventricular tachycardia, they could be certain of the diagnosis from the conventional electrocardiogram in only two instances, whereas they could make the diagnosis in all 10 clinically. P waves are usually best seen in lead V1, but resort may have to be made to special leads for their identification, e.g. CR1, S5*, or œsophageal leads. The diagnosis of phasic aberrant ventricular conduction in the presence of paroxysmal tachycardia is facilitated if a record of the beginning of a paroxysm is available as the presence of a $P$ wave before the first aberrant QRS complex favours a supraventricular tachycardia (Fig. 7 and 8). Furthermore, a progressive increase in the aberrancy of the QRS complexes as the rate speeds up favours phasic aberrant ventricular conduction (Fig. 7).

Recognition of Phasic Aberrant Ventricular Conduction in Presence of Atrial Fibrillation. As the recognition of phasic aberrant ventricular conduction is dependent upon the presence of $P$ waves before the aberrant QRS complexes, its diagnosis during atrial fibrillation is extremely difficult and indeed cannot be made with certainty. However, the following features may point to its presence.

(a) Average ventricular rate. As prematurity favours aberration, phasic aberrant ventricular conduction tends to occur with relatively fast average ventricular rates (Fig. 9): ventricular extrasystoles tend to occur with slow average ventricular rates.

(b) The QRS pattern. Phasic aberrant ventricular conduction usually results in a right bundlebranch block pattern (Fig. 2, 3, and 9), whereas ventricular extrasystoles show left or right bundlebranch block patterns with equal frequency. In atrial fibrillation the rapid supraventricular discharges occur at all stages of the cardiac cycle and find the conducting system in different phases of recovery: this results in varying degrees of aberration and hence frequent variation in the shape of the bizarre QRS complexes. Ventricular extrasystoles usually have one or two fixed patterns.

(c) The coupling interval. Ventricular extrasystoles of identical contour nearly always occur at a fixed interval from the preceding beat, i.e. they have fixed coupling. Beats conducted with ventricular aberration show no such relation and occur at varying intervals following the preceding beat.

(d) The attempt at a compensatory pause. Ventricular extrasystoles are usually followed by a relatively long pause, i.e. there is an attempt at a compensatory pause: this is because the ectopic ventricular impulse penetrates the $\mathrm{A}-\mathrm{V}$ node retrogradely and renders it refractory to an early succeeding impulse. With phasic aberrant ventricular conduction there is no attempt at a compensatory pause and the bizarre complex may thus be followed by a relatively short cycle (Fig. 8).

\footnotetext{
* The selector switch is set at S1; the right arm-negative-electrode is placed over the manubrium, and the left arm-positive-electrode is placed over the right fifth interspace adjacent to the sternum.
} 


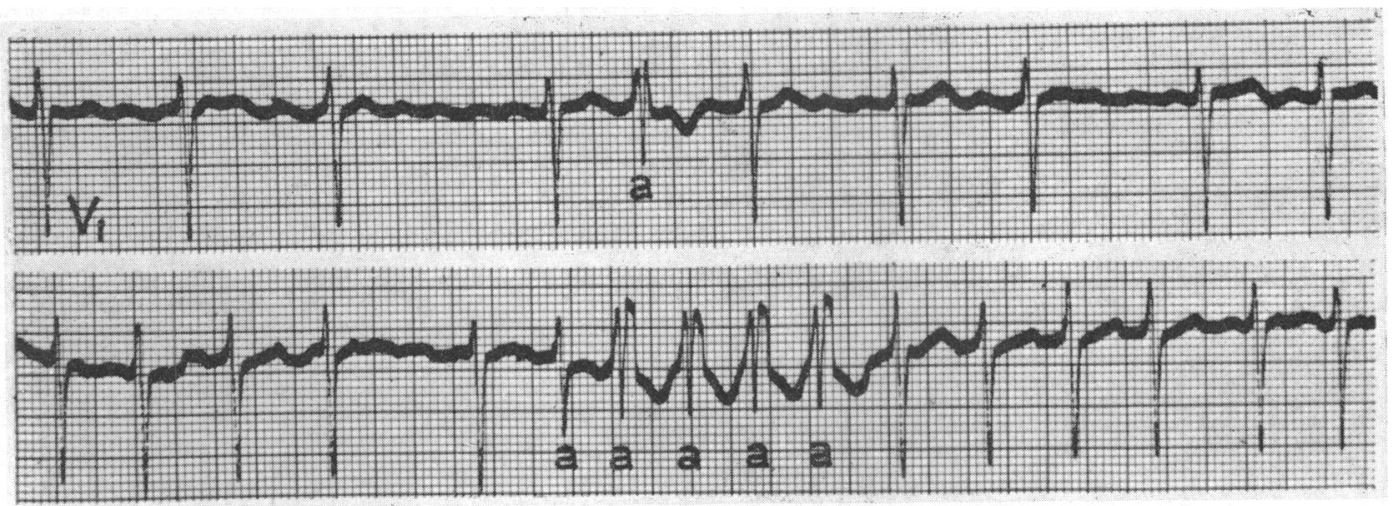

FIG. 9.-Electrocardiogram (continuous strip of lead V1) showing atrial fibrillation with anomalous beats (labelled a). These anomalous beats are probably due to aberrant ventricular conduction as: (1) there is no attempt at a compensatory pause; (2) there is slight variation in shape between the first and second anomalous beats in the lower strip; (3) there is a tendency to a fast rhythm; (4) the coupling intervals vary; and (5) the anomalous pattern is that of right bundle-branch block. Note also that the anomalous beat is an early beat following a long $\mathbf{R}-\mathbf{R}$ interval.

Note. As phasic aberrant ventricular conduction is favoured by a long preceding $\mathbf{R}-\mathbf{R}$ interval, it will most likely occur when a relatively short cycle follows a relatively long one (Fig. 8). However, the precipitation of a ventricular extrasystole is also favoured by a long preceding $R-R$ interval-the rule of bigeminy (Langendorf, Pick, and Winternitz, 1955). The long-short sequence is therefore of no value in differentiating the two conditions.

\section{Significance of Phasic Aberrant Ventricular Conduction}

The awareness that one or a succession of bizarre QRS complexes is not necessarily due to ectopic ventricular rhythm but may be due to phasic aberrant ventricular conduction is of major clinical import. Thus, digitalis is the treatment of choice in atrial fibrillation with rapid ventricular reresponse: it may, however, be withheld if the tracing is interspersed with bizarre QRS complexes, due to phasic aberrant ventricular conduction, that are incorrectly diagnosed as ventricular extrasystoles. Indeed, phasic aberrant ventricular conduction is usually an indication to increase digitalis therapy whereas ectopic ventricular rhythm is an indication to discontinue digitalis therapy or to proceed with caution. It is possible that some of the cases of "ventricular tachycardia" that have responded to digitalis may be accounted for by the failure to recognize phasic aberrant ventricular conduction. Furthermore, it may also, in part, explain the statement that ventricular extrasystoles are more likely to be seen in the presence of atrial fibrillation.

Phasic aberrant ventricular conduction is usually associated with heart disease although its incidence and prognostic import relative to normal A-V conduction has still be be elucidated. Nevertheless its recognition and distinction from ectopic ventricular rhythms is extremely important with regard to prognosis. Phasic aberrant ventricular conduction probably has the same prognostic import as the equivalent supraventricular rhythm with normal $\mathrm{A}-\mathrm{V}$ conduction and thus does not necessarily connote the adverse prognosis usually associated with ectopic ventricular rhythms.

\section{SUMMARY}

The diagnostic features and mechanisms of the temporary, intermittent, or isolated forms of aberrant ventricular conduction are discussed and their clinical and prognostic import stressed. The term phasic aberrant ventricular conduction is suggested for these forms to distinguish them from the established or non-phasic forms of aberrant ventricular conduction. The differentiation of phasic aberrant ventricular conduction and ectopic ventricular rhythms is described. 
We wish to thank Dr. I. Frack, Superintendent of Baragwanath Hospital, for permission to publish this report. We are indebted to the photographic department of the University of the Witwatersrand, Medical School, for the photographic reproductions. We would like to thank Dr. H. J. L. Marriott for his kind permission to reproduce Fig. 8 and 9.

\section{REFERENCES}

Gouaux, J. L., and Ashman, R. (1947). Amer. Heart J., 34, 366.

Langendorf, R. (1950). Exp. Med. Surg., 8, 228.

(1951). Amer. Heart J., 41, 700.

, Pick, A., and Winternitz, M. (1955). Circulation, 11, 422.

Lewis, T. (1910). Heart, 1, 262.

(1912). Heart, 3, 279.

Drury, A. N., and Bulger, H. A. (1921). Heart, 8, 83.

- and Master, A. M. (1925). Heart, 12, 209.

Mines, G. R. (1913). J. Physiol (Lond.), 46, 349.

Schamroth, L. (1960). Amer. Heart J., 59, 506.

Scherf, D. (1927). Wien. Arch. inn. Med., 14, 443.

-

Schrire, V., and Vogelpoel, L. (1955). Amer. Heart J., 49, 162.

Shearn, M. A., and Rytand, D. A. (1953). Arch. intern. Med., 91, 448.

Trendelenburg, W. (1903). Arch. Anat. Physiol., Lpz., Physiol. Abt., 311.

Vesell, H. (1941). Amer. J. med. Sci., 202, 198. 